\title{
Knockout of microRNA-26a promotes lung development and pulmonary surfactant synthesis
}

\author{
YI-FAN SUN*, QING KAN*, YANG YANG, YING-HUI ZHANG, JIN-XIN SHEN, CUN ZHANG and XIAO-YU ZHOU \\ Department of Neonatology, Children's Hospital of Nanjing Medical University, Nanjing, Jiangsu 210008, P.R. China
}

Received August 10, 2017; Accepted January 22, 2018

DOI: $10.3892 / \mathrm{mmr} .2018 .8602$

\begin{abstract}
Normal formation and function of the lungs are essential for the transition of the fetus to an air-breathing environment at birth. The synthesis of pulmonary surfactant (PS), which is produced by type II alveolar epithelial cells (AECIIs), is required for proper lung development. Previous in vitro studies have suggested that PS synthesis is regulated by microRNA (miR)-26a in fetal rat AECIIs. The present study explored the potential role of miR-26a in lung development and PS synthesis by using a miR-26a-1/miR-26a-2 double knockout mouse model. Hematoxylin and eosin staining and transmission electron microscopy were used to observe the morphology of fetal lungs. Reverse transcription-quantitative polymerase chain reaction and western blot analysis were performed to examine the mRNA and protein levels of surfactant-associated proteins. The results demonstrated that the lung formation in the knockout mice was more mature, and that there were more mature lamellar bodies inside AECIIs in miR-26a knockout mice at late stages of lung development. The findings further demonstrated that knockout of miR-26a increased surfactant-associated mRNA and protein expression levels. The results indicated that knockout of miR-26a promotes lung development and PS synthesis.
\end{abstract}

\section{Introduction}

In mammals, the transition of the fetus from an aqueous to an air breathing environment at birth is dependent upon the formation and function of the lungs. Neonatal respiratory distress, primarily resulting from the immaturity of fetal lungs is a common cause of morbidity and mortality in preterm infants (1). Synthesis and metabolism of pulmonary surfactant

Correspondence to: $\mathrm{Dr}$ Xiao-Yu Zhou, Department of Neonatology, Children's Hospital of Nanjing Medical University, 72 Guangzhou Road, Nanjing, Jiangsu 210008, P.R. China

E-mail: xyzhou161@163.com

*Contributed equally

Key words: microRNA-26a, knockout mice, lung development, pulmonary surfactant, type II alveolar epithelial cells
(PS) in type II alveolar epithelial cells (AECIIs) is closely associated with the state of maturation of the fetal lung $(2,3)$. PS is a complex lipid and protein mixture, produced by AECIIs from late fetal development onwards, and prevents alveolar collapse by regulating surface tension at the pulmonary air-liquid interface (4). It is composed of $90 \%$ lipids and $10 \%$ surfactant-associated proteins, including surfactant protein (SP)-A, SP-B, SP-C and SP-D. SP-A and SP-D, hydrophilic collectin proteins, participate in pulmonary host defense and modify immune responses, whereas SP-B and SP-C, small hydrophobic proteins, together with dipalmitoylphosphatidylcholine (DPPC), confer surface tension lowering properties to the material (5-7). MicroRNAs (miRNAs/miRs) are a class of small non-coding RNAs that suppress gene translation, most commonly through promoting mRNA degradation or disrupting mRNA translation. A total of $>100$ miRNAs have been identified to be crucial during lung development, however detailed functions of the majority of them are unknown (8). The authors previously demonstrated that miR-26a was one of seven miRNAs that demonstrated significant alterations in expression, as determined by miRNA profiling, at three-time points in the developing rat lung (9). It was also identified that PS synthesis is regulated by miR-26a in fetal rat AECIIs (2). However, there is a lack of data regarding exactly how miR-26a carries out its specific function in vitro and in transgenic animal studies.

As the lung development and PS regulatory processes are quite well conserved, the mouse is an ideal animal to study the stages of lung development (10-12). Lung development in mouse begins at pseudoglandular stage (E9.5-E16.5). This is followed by the canalicular (E16.5-E17.5) and saccular [E18.5-postnatal day (P) 5] stages, during which these terminal branches narrow and form clusters of epithelial sacs that later develop into alveoli in preparation for respiration at birth. Finally, full maturation of the alveolus occurs during the alveolarization stage (P0-P14) (8). The clustered regularly interspaced short palindromic repeat/CRISPR-associated protein 9 (CRISPR/Cas9) system is a useful gene knockout technique for investigating gene function in vivo; since its development, this technique has been utilized to generate knockout cell lines $(13,14)$ and animal models $(15,16)$. An miR-26a-1/miR-26a-2 double knockout mouse model has been successfully obtained by using the CRISPR/Cas9 system (17). The present study used the miR-26a double knockout mouse model to investigate the role of miR-26a in different stages of 
lung development and PS synthesis in in vitro and transgenic animal studies.

\section{Materials and methods}

Animals. C57BL/6J (25 female, weight 11 \pm 2 g, 3-4 weeks old and 3 male, weight $27 \pm 2 \mathrm{~g}, 13$ weeks old) and FVB mice (12 female, weight $20 \pm 2$ g, 7-8 weeks old) were purchased from Nanjing Biomedical Research Institute of Nanjing University, China. All the mice were housed apart and were maintained on a normal 12-h light/dark cycle at a temperature of $20-26^{\circ} \mathrm{C}$ ) and $40-70 \%$ humidity. Water and food was supplied ad libitum. The housing conditions met the specific pathogen-free (SPF) standards. Lungs were obtained at gestational days (E) 16.5 and 18.5, and P day 2 from double knockout mice and wild-type mice. The pregnant mice were killed by cervical dislocation after anesthesia with $2 \%$ chloral hydrate $(0.2 \mathrm{ml} / 10 \mathrm{~g})$. Fetal mice were removed and fixed to a clean operating table. Lungs were dissected free of heart and trachea, and placed into EP tubes. All procedures were approved by the Nanjing Medical University Animal Ethical and Welfare Committee (Nanjing, China).

Microinjection. A Cas9 expression plasmid (GenScript Biotech Corporation, Piscataway Township, NJ, USA) containing the SP6 promoter was used as a template for in vitro transcription (IVT) using an mMESSAGE mMACHINE SP6 kit (Invitrogen; Thermo Fisher Scientific, Inc., Waltham, MA, USA) following linearization and purification. As previously described (15), fusion crRNA and tracrRNA expression vectors were constructed with a customizable synthetic (sg)RNA template using miR-26a-1F/R and miR-26a-2F/R primers (Table I). The T7-sgRNA polymerase chain reaction (PCR) product was purified and used as a template for IVT, performed using a T7 kit (Takara Bio, Inc., Otsu, Japan). Female C57BL/6J mice were superovulated using pregnant mare serum gonadotropin (GenWay Biotech Inc., San Diego, CA, USA) and were injected with human chorionic gonadotropin (GenWay Biotech Inc.) following $48 \mathrm{~h}$. The superovulated female mice were mated with C57BL/6J stud males, and fertilized embryos were collected from their oviducts. miR-26a-1 and miR-26a-2 sgRNAs (12.5 ng/ $\mu \mathrm{l}$ each) were mixed with Cas9 mRNA (25 ng/ $\mu \mathrm{l})$, and the mixture was microinjected into the cytoplasm of the C57BL/6J mouse embryos at the pronuclei stage. The injected zygotes were cultured in KSOM (EMD Millipore, Billerica, MA, USA) with amino acids at $37^{\circ} \mathrm{C}$ and $5 \% \mathrm{CO}_{2}$ in air until the blastocyst stage (3.5 days). Subsequently, $\sim 15-25$ blastocysts were transferred into the uterus of each pseudopregnant FVB female.

Genotyping and breeding. Following 19 days, DNA was extracted from the pups' tails (founder mice), and the mice were genotyped by PCR and sequencing. Then, male (7-week-old) and female (4-week-old) founder mice were mated with wild-type mice. The next generation of mice (F1 mice) was obtained and genotyped until mice positive for either or both mutations in miR-26a-1 and/or miR-26a-2 were identified, indicating the successful generation of gene knockout mouse strains. Positive mice ( $>3$ females and $>3$ males) were mated with wild-type mice (SPF standard), and the PCR products from the tail DNA samples were identified following restriction digestion with $\mathrm{T} 7$ endonuclease I (T7EI) and resolution in a $1 \%$ agarose gel. The $20 \mu \mathrm{l}$ PCR mixture contained $10 \mu \mathrm{l}$ master mix (Vazyme Biotech, Nanjing, China), $8.2 \mu \mathrm{l}$ $\mathrm{ddH}_{2} \mathrm{O}, 1 \mu \mathrm{l}$ DNA, and $0.4 \mu \mathrm{l}$ each of the miR-26a-1 forward and reverse primers or $0.4 \mu \mathrm{l}$ each of the miR-26a- 2 forward and reverse primers (Table I). The PCR amplification program was as follows: $94^{\circ} \mathrm{C}$ for $5 \mathrm{~min} ; 35$ cycles at $94^{\circ} \mathrm{C}$ for $30 \mathrm{sec}$, $55^{\circ} \mathrm{C}$ for $30 \mathrm{sec}$, and $72^{\circ} \mathrm{C}$ for $60 \mathrm{sec}$; and $72^{\circ} \mathrm{C}$ for $7 \mathrm{~min}$. The amplicons were separated by $1.0 \%$ agarose gel electrophoresis. The bands were visualized by ultraviolet projection and a Gel Doc XR+ Imaging System with Image Lab Software version 5.2.1 (Bio-Rad Laboratories, Inc., Hercules, CA, USA).

Morphological analysis. Lung tissue was fixed with $4 \%$ buffered paraformaldehyde at $4^{\circ} \mathrm{C}$ overnight, dehydrated and embedded in paraffin. Sections at 3-4 $\mu \mathrm{m}$ thickness were prepared for hematoxylin and eosin (H\&E) staining and immunohistochemistry. Sections were dewaxed with xylene and hydrated in a series of ethanol/water solutions. Then, the sections were stained with hematoxylin for $5 \mathrm{~min}$, differentiated with $1 \%$ ethanol hydrochloride for $3 \mathrm{sec}$ and transferred to eosin solution for $2 \mathrm{~min}$ at room temperature. Then the sections were dehydrated and mounted. Analysis was conducted under a microscope (BX51; Olympus Corporation, Tokyo, Japan). The sections pretreated for immunohistochemistry were dewaxed and rehydrated with xylene and ethanol/water. The sections were immersed in sodium citrate antigen retrieval solution (maintained at a sub-boiling temperature for $8 \mathrm{~min}$, standing for $8 \mathrm{~min}$ and then followed by another sub-boiling temperature for $7 \mathrm{~min}$ ). To block endogenous peroxidase, sections were immersed in $3 \% \mathrm{H}_{2} \mathrm{O}_{2}$, kept in a dark place and incubated at room temperature for $15 \mathrm{~min}$. Then, sections were blocked with $3 \% \mathrm{BSA}$ at room temperature for $30 \mathrm{~min}$. The sections were incubated at $4^{\circ} \mathrm{C}$ overnight with primary antibodies, rinsed with PBS, incubated with HRP-conjugated goat-anti-rabbit secondary antibody (K5007; Dako; Agilent Technologies, Inc., Santa Clara, CA, USA), and developed using diaminobenzidine. Subsequently, Hematoxylin staining solution was used for counterstaining the nucleus. Finally, the sections were dehydrated and mounted. Analysis was conducted under a microscope (BX51; Olympus Corporation). Primary antibodies used included rabbit anti-SP-A antibody (ab115791; 1:100; Abcam, Cambridge, MA, USA), rabbit anti-pro and mature SP-B antibody (ab40876; 1:200; Abcam), rabbit anti-pro-SP-C antibody (AB3786; 1:1,000; EMD Millipore).

Electron microscopy was performed on lung tissues obtained from E16.5, E18.5 and P2 mutant mice and littermate controls. Lungs were fixed in glutaraldehyde at $4^{\circ} \mathrm{C}$ for $2-4 \mathrm{~h}$, post-fixed with $1 \% \mathrm{OsO}_{4}$ in $\mathrm{PBS}$ for $2 \mathrm{~h}$ at room temperature, dehydrated with ethanol/water and acetone, infiltrated and embedded by acetone and EMBed 812 (90529-77-4; SPI Supplies, West Chester, PA, USA), heated in a $60^{\circ} \mathrm{C}$ oven for $48 \mathrm{~h}$. Sections of 60-80 $\mathrm{nm}$ thickness were prepared using an ultramicrotome, stained with uranyl acetate in pure ethanol for $15 \mathrm{~min}$ and lead citrate for $15 \mathrm{~min}$, and dried overnight at room temperature. Finally, electron microscopy (Hitachi, Ltd., Tokyo, Japan) was performed.

$R N A$ extraction and reverse transcription-quantitative PCR $(R T-q P C R)$. Total RNA was extracted from lung tissues 
Table I. Customizable synthetic RNA primer sequences.

\begin{tabular}{ll}
\hline Variables & \multicolumn{1}{c}{ Sequence (5'-3') } \\
\hline $\begin{array}{l}\text { mmu-mir-26a-1 } \\
\text { Forward }\end{array}$ & GGGCTCTTTCCTTAGACTTGG \\
Reverse & GACCTGCTTTGCTCATAACACTC \\
mmu-mir-26a-2 & \\
Forward & GTTGGTGCTGATGTGGGCTAG \\
Reverse & CTGGGAGACAGAGTGGATTGC
\end{tabular}

mir, microRNA.

according to the manufacturer's protocol using TRIzol ${ }^{\circledR}$ reagent (Thermo Fisher Scientific, Inc.). The RNA quantity control and concentration were detected by NanoDrop 2000 Spectrophotometer (Thermo Fisher Scientific, Inc.). A High-Capacity cDNA Reverse Transcription kit (Applied Biosystems; Thermo Fisher Scientific, Inc.) was used for RT. qPCR was performed using SYBR Green (Roche, Shanghai, China), and specific primers for SP-A (Sftpa1), SP-B (Sftpb), and SP-C (Sftpc) were synthesized according to published cDNA sequences (Table II). The PCR was performed with an ABI 7500 thermal cycler (Applied Biosystems; Thermo Fisher Scientific, Inc.) and the reaction conditions were $95^{\circ} \mathrm{C}$ for $10 \mathrm{~min}, 50^{\circ} \mathrm{C}$ for $2 \mathrm{~min}$, then 40 cycles at $60^{\circ} \mathrm{C}$ for $1 \mathrm{~min}$, and $95^{\circ} \mathrm{C}$ for $15 \mathrm{sec}$. Dissociation curves were generated for genes under the following conditions: $95^{\circ} \mathrm{C}$ for $15 \mathrm{sec}, 60^{\circ} \mathrm{C}$ for $60 \mathrm{sec}$, and $95^{\circ} \mathrm{C}$ for $15 \mathrm{sec}$. Relative quantification of gene expression in multiple samples was achieved by normalization against an endogenous control, GAPDH. Then, the relative expression levels were compared between the knockout mice and wild-type mice using the $2^{-\Delta \Delta C q}$ method (18).

Protein extraction and western blot analysis. Briefly, total proteins from tissues were extracted using radioimmunoprecipitation assay buffer (Beyotime Institute of Biotechnology, Nantong, China) containing protease inhibitors and the concentrations were measured by bicinchoninic acid (BCA) solution (Beyotime Institute of Biotechnology, Nantong, China). The western blotting was performed under the standard program. Proteins $(60 \mu \mathrm{g} / \mathrm{lane})$ were loaded on $12 \%$ SDS-PAGE gels, and the separated proteins were transferred onto PVDF membrane (EMD Millipore). The membrane was then blocked for non-specific binding for $2 \mathrm{~h}$ by incubating with $5 \%$ fat-free dry milk in $100 \mathrm{mM}$ Tris-buffered saline plus $0.1 \%$ Tween-18 (TBS-T). Primary antibodies were diluted in blocking buffer and incubated with membrane overnight at $4^{\circ} \mathrm{C}$. Primary antibodies used included rabbit anti-SP-A antibody (ab115791; 1:4,000; Abcam), rabbit anti-pro and mature SP-B antibody (ab40876; 1:5,000; Abcam), rabbit anti-pro-SP-C antibody (AB3786; 1:500; EMD Millipore), and rabbit anti-GAPDH antibody (KGAA002; 1:1,000; Nanjing KeyGen Biotech Co., Ltd., Nanjing, China). Following washing in TBST 3 times, the membrane was incubated with HRP-conjugated goat-anti-rabbit secondary antibody (bs-0295G-HRP; 1:2,000; BIOSS, Beijing, China) for $1.5 \mathrm{~h}$ at room temperature. The antibody-detected protein bands were visualized by an
Table II. Reverse transcription-quantitative polymerase chain reaction primer sequences.

\begin{tabular}{lc}
\hline Variables & Sequence (5'-3') \\
\hline m-SP-A & \\
Forward & ACCTCCTTCTGCTTGGAACC \\
Reverse & CCAGGAGCAGGCACTTTCTA \\
m-SP-B & \\
Forward & CTGCTTCCTACCCTCTGCTG \\
Reverse & ATCCTCACACTCTTGGCACA \\
m-SP-C & \\
Forward & TTGTCGTGGTGATTGTAGGG \\
Reverse & AGGTAGCGATGGTGTCTGCT \\
m-GAPDH & \\
Forward & GGTGAAGGTCGGTGTGAACG \\
Reverse & CTCGCTCCTGGAAGATGGTG
\end{tabular}

SP, surfactant protein.

enhanced chemiluminescence detection system (ChemiDoc XRS + Imaging System; Bio-Rad Laboratories, Inc.)

Statistical analysis. All quantitative data were expressed as the mean \pm standard error of the mean. All experiments were repeated 3 times, and data represent consistent results. The results were analyzed using the Student's t-test with SPSS software, version 17.0 (SPSS, Inc., Chicago, IL, USA) and GraphPad Prism 5.0 (GraphPad Software, Inc., La Jolla, CA, USA). $\mathrm{P}<0.05$ was considered to indicate a statistically significant difference.

\section{Results}

Successful establishment of miR-26a-1/miR-26a-2 double knockout mouse model. CRISPR/Cas9 plasmids targeting miR-26a-1 and miR-26a-2 were constructed. Mice harboring mutations in the two genes were produced by coinjection of Cas9 with miR-26a-1 and miR-26a-2 sgRNAs into zygotes. The efficiency of obtaining mice carrying mutations in the two targeted genes reached $28 \%$, and $~ 56 \%$ of the mice harbored a biallelic mutation in one of the targeted genes (17). Phenotypic analysis revealed that the majority of the double knockout mice did not demonstrate any differences in phenotype, body weight, pregnancy rate, birth rate, or growth rate compared with the wild-type mice (17). A number of the knockout mice were blind and had white abdominal hair, however the reason was unknown and requires further investigation.

Knockout of miR-26a promotes maturation in lung formation. $\mathrm{H} \& \mathrm{E}$ staining was performed to observe differences in lung formation at E16.5, E18.5, and P2 between the miR-26a double knockout mice and wild-type mice. At the beginning of the canalicular stage, numerous epithelial tubules were observed, and the high columnar epithelial cells altered to a low cuboidal shape in lungs from the two groups, however dilated lumens and a more prominent aerated area was observed in the 
A $\quad \mathbf{E} 16.5 \quad$ WT(x400)

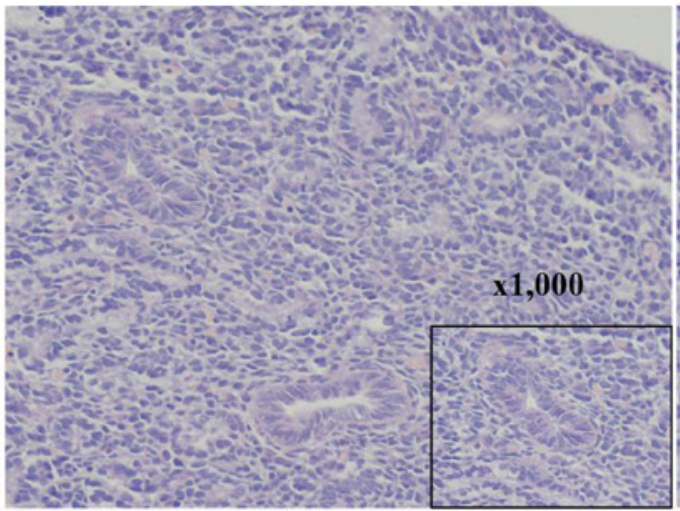

B $\quad$ E18.5 WT(x200)

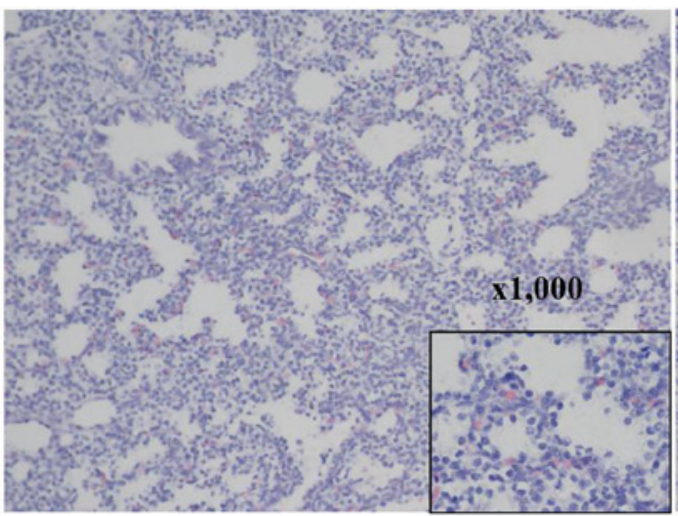

C P2

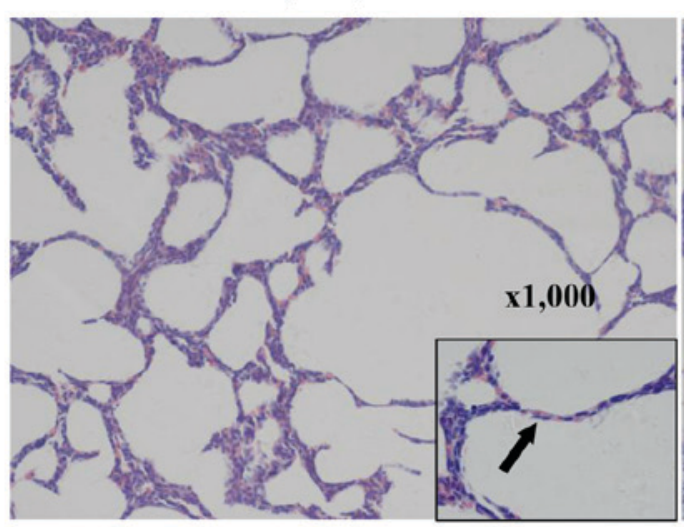

WT(x200)

D

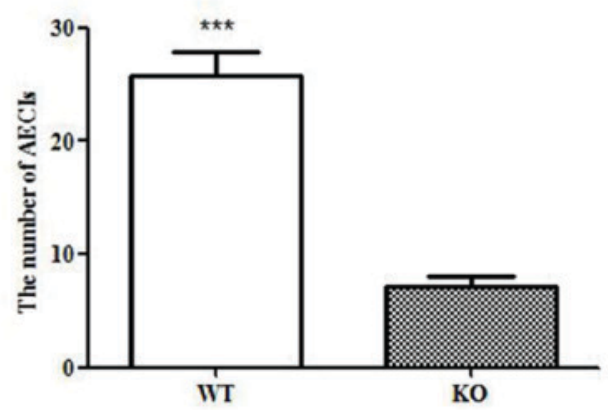

\section{KO(x400)}

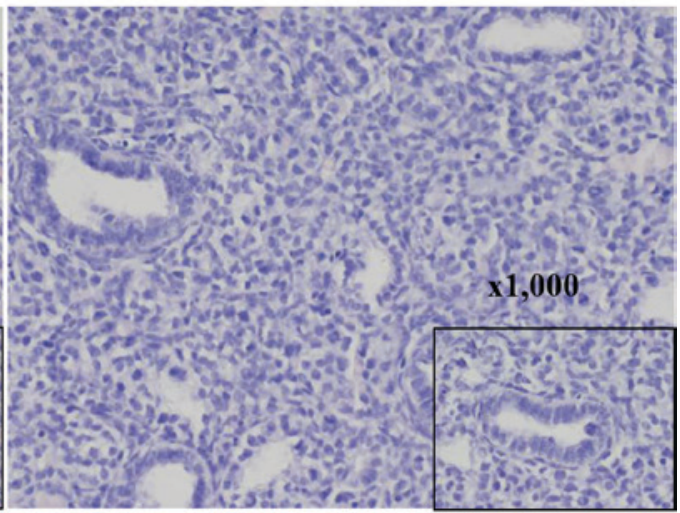

$\mathbf{K O}(\mathbf{x 2 0 0 )}$

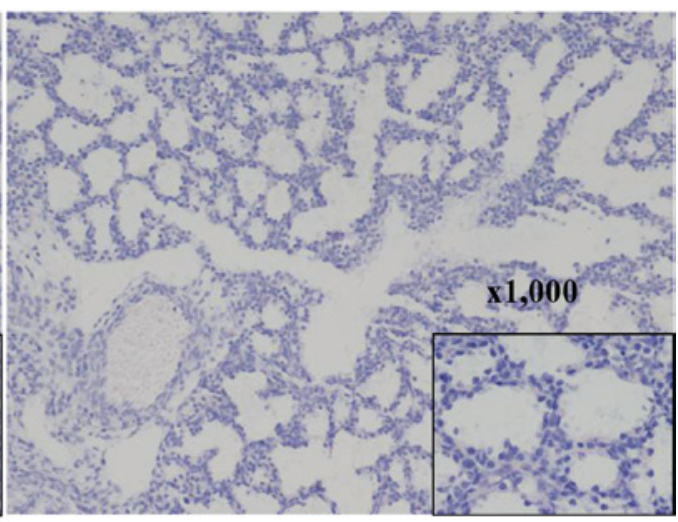

$\mathbf{K O}(\mathbf{x 2 0 0 )}$
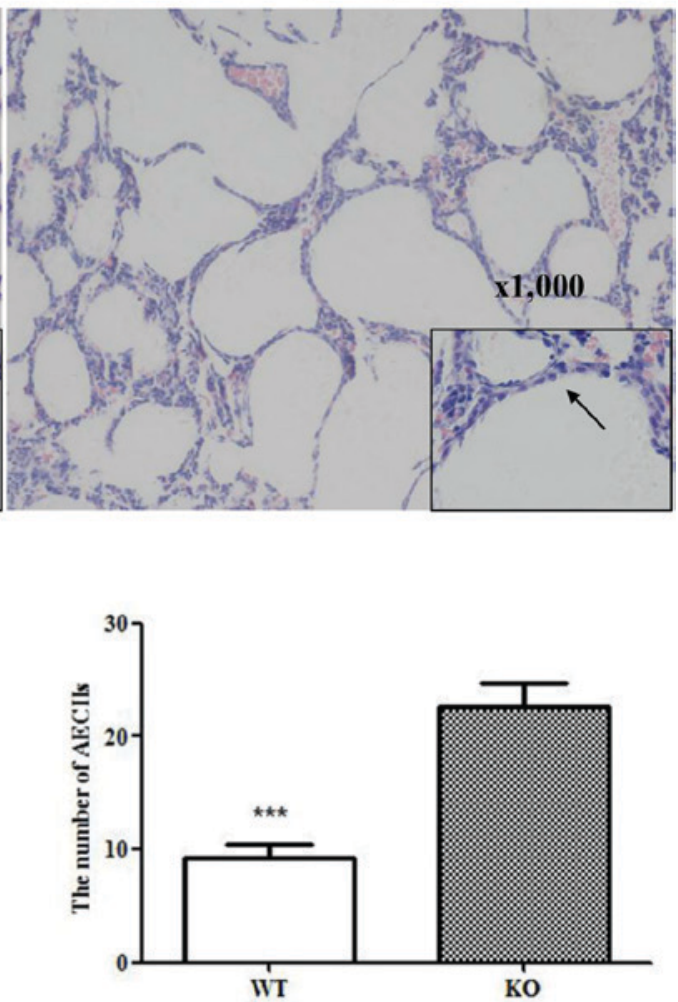

Figure 1. Effects of miR-26a on maturation of lung formation, observed by Hematoxylin and eosin staining (n=3). (A) At an early stage (E16.5), dilated lumens and a prominent aerated area were observed in lungs of KO mice compared with WT mice (magnification, $\mathrm{x} 400$ and x1,000). (B) Numerous AECIIs were produced (magnification, $\mathrm{x} 1,000$ ) and the alveolar structure of $\mathrm{KO}$ mice lungs was more mature compared with WT mice lungs at E18.5 (magnification, $\mathrm{x} 200$ ). (C) Although lung tissues from the two groups demonstrated further alveolar maturation (magnification, x200), more AECIIs (fine arrows) and fewer AECIs (thick arrows) were detected in KO mice compared with WT mice (magnification, x1,000) and (D) the difference was statistically significant. Data are presented as the mean \pm standard deviation ( $\mathrm{n}=6,{ }^{* * *} \mathrm{P}<0.001 \mathrm{vs}$. KO mice). miR, microRNA; AEC, alveolar epithelial cell; KO, knockout mice; WT, wild-type mice; P, postnatal day. 
A $\quad \mathbf{E} 16.5$ WT $(\mathbf{x 2 , 0 0 0 )}$ KO $(\mathbf{x 2 , 0 0 0 )}$

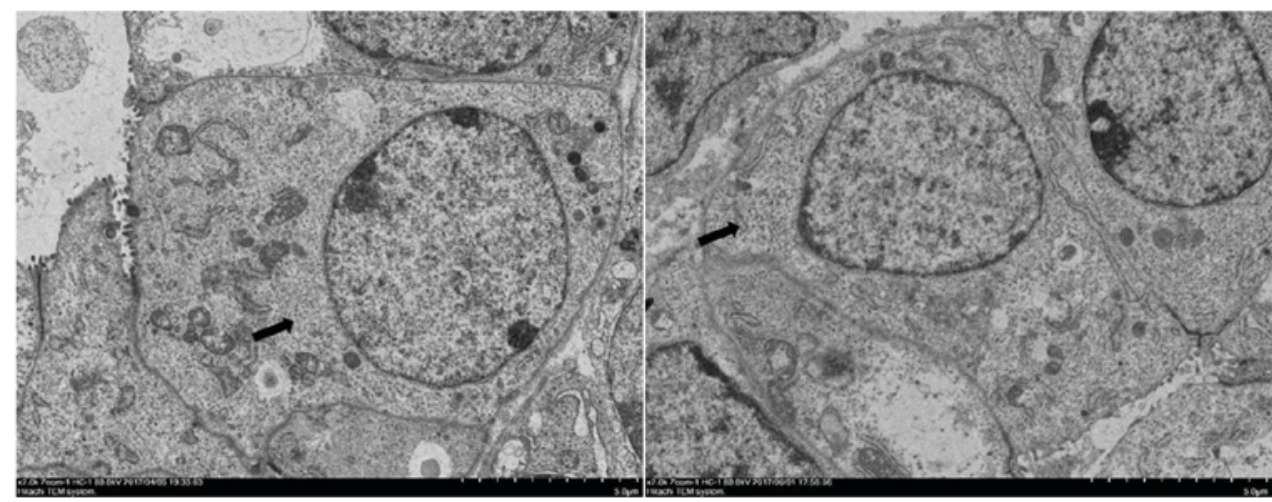

B $\quad$ E18.5 WT $(x 2,000)$

KO $(\mathbf{x} 2,000)$

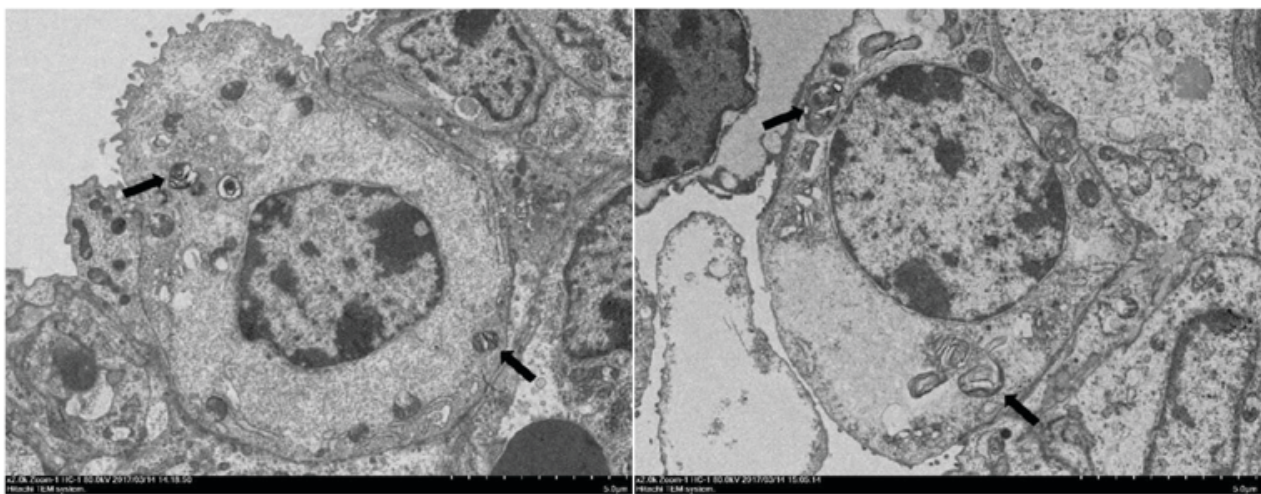

C P2 WT $(x 2,500)$

KO $(\mathbf{x 2 , 5 0 0 )}$

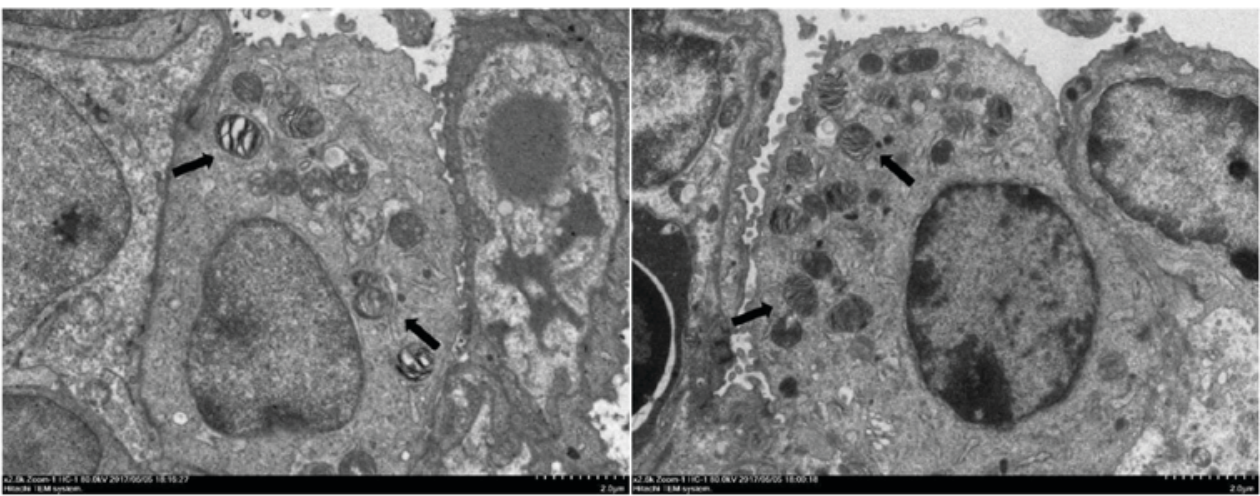

Figure 2. Importance of miR-26a in the production process of PS was detected by transmission electron microscopy ( $\mathrm{n}=3$ ). (A) Increased intracellular glucogen (arrows) was observed in KO mice lungs compared with WT mice lungs at E16.5 (magnification, x2,000). (B and C) Increased mature lamellar bodies (arrows) inside AECIIs were detected in the KO mice compared with the WT mice at E18.5 (magnification, x2,000) and P2 (magnification, x2,500). PS, pulmonary surfactant; miR, microRNA; KO, knockout mice; WT, wild-type mice; AEC, alveolar epithelial cell; P, postnatal day.

knockout mice lungs (E16.5; Fig. 1A). At the latter stage (E18.5; Fig. 1B), a large number of AECIIs were produced and lungs from miR-26a knockout mice demonstrated an increase in the maturation of alveolar structure compared with wild-type mice lungs. Lung tissues from the two groups demonstrated further alveolar maturation characterized by further-expanded alveolar air spaces and thinning air sac wall at P2, however more AECIIs and fewer type I alveolar epithelial cells (AECIs) were observed in knockout mice lungs (Fig. 1C and D). These observations indicated increased maturity of lung tissues from the double knockout mice in lung development.

miR-26a serves an important role in the production process of $P S$. Compared with wild-type mice lungs, the epithelial cells of knockout mice lungs had more glucogen at the beginning of the canalicular stage (E16.5, Fig. 2A). PS production and secretion was increasingly detected in knockout mice lungs at E18.5d and P2, as demonstrated by more mature lamellar bodies inside AECIIs (Fig. 2B and C).

Knockout of miR-26a increases the expression of $S P-A, S P-B$ and $S P-C$. To explore the effect of miR-26a on PS synthesis, the expression of SP-A, SP-B and SP-C were examined by immunohistochemistry, RT-qPCR and western blot analysis. The SP-A, SP-B and SP-C protein levels, detected by immunohistochemistry at E18.5 and P2, were significantly increased in knockout mice compared with the wild-type mice (Fig. 3A-C). Results consistent with immunohistochemistry were obtained 
A

SPA

SPB

SPC

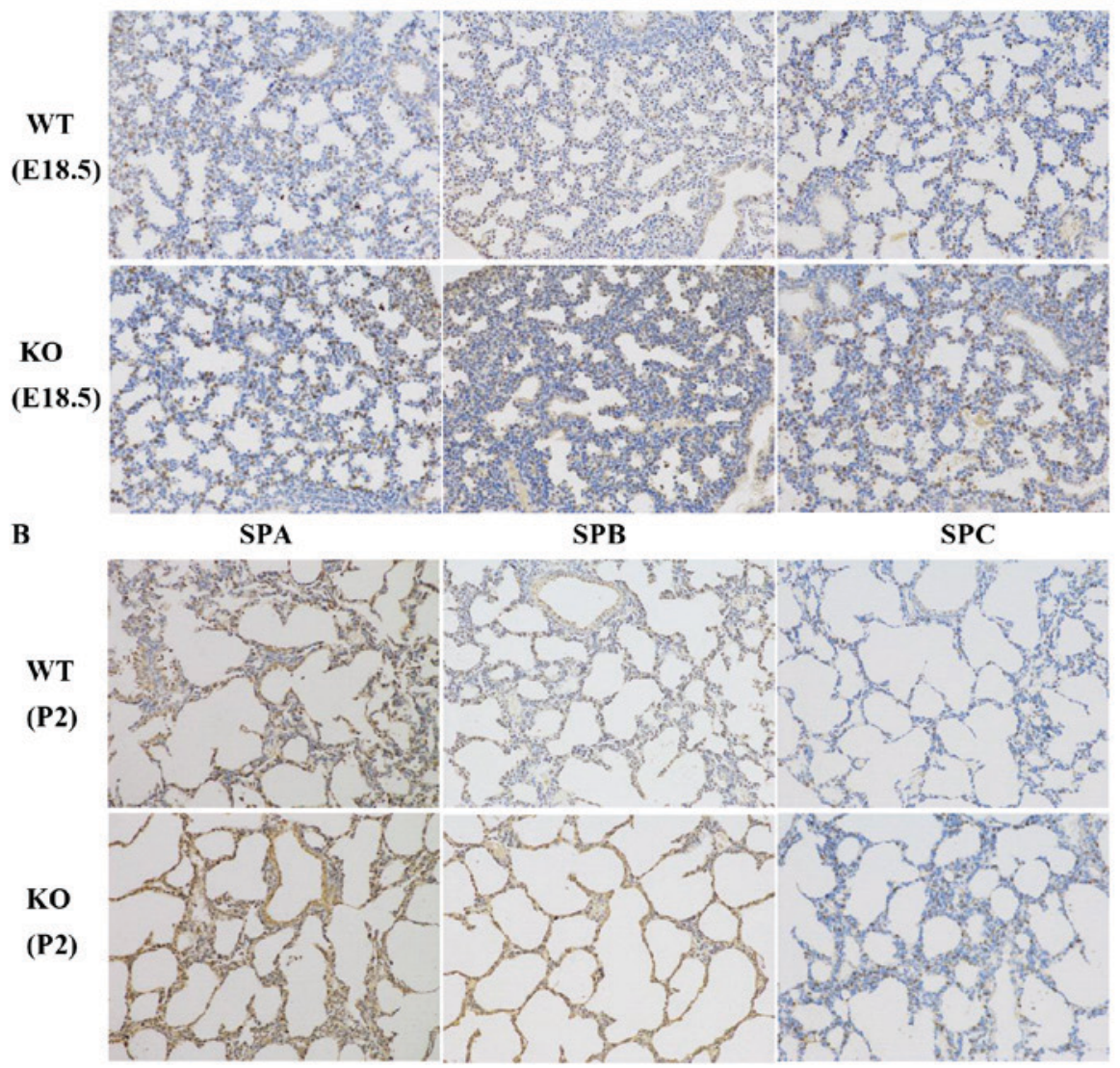

C $\quad \mathbf{E} 18.5$

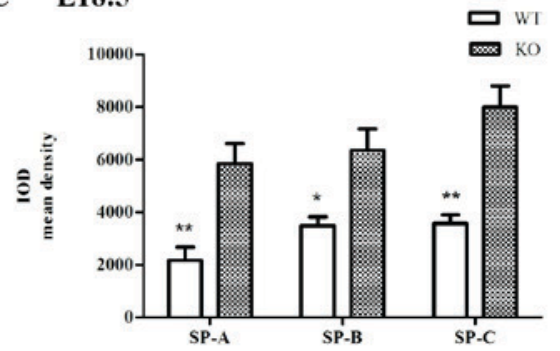

P2

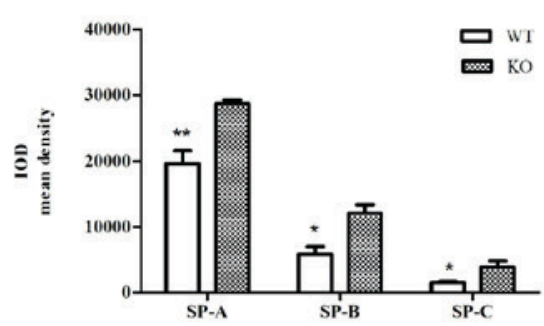

D $\quad$ E18.5 SPA

SPB

SPC

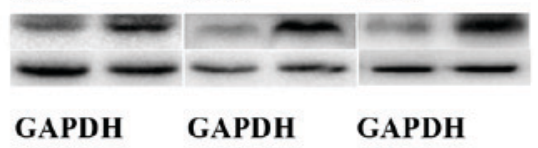

E $\quad$ E18.5

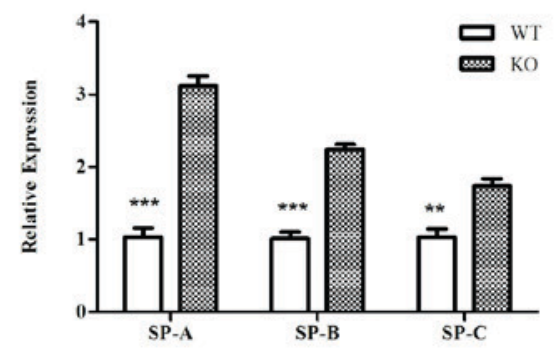

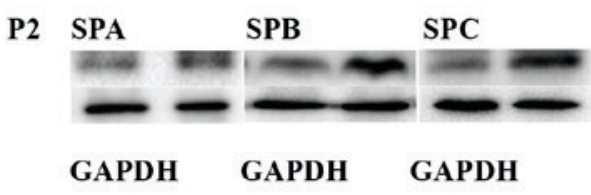

P2

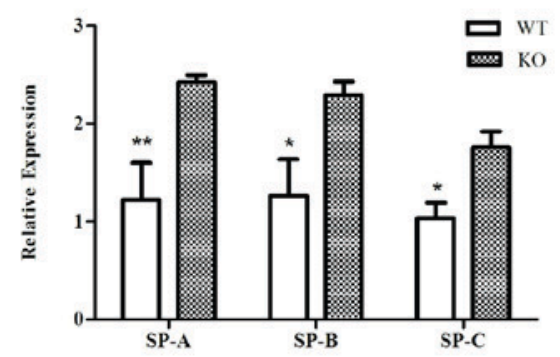

Figure 3. Effects of miR-26a on the expression of SP-A, SP-B, and SP-C. (A and B) SP-A, SP-B and SP-C were identified by immunohistochemistry (representative images; magnification, x200). (C) Analysis of the mean IOD demonstrated that the SP-A, SP-B and SP-C protein levels were significantly increased in the KO mice at E18.5 and P2. Data are presented as the mean \pm standard deviation $\left(\mathrm{n}=4 ;{ }^{*} \mathrm{P}<0.05 ;{ }^{* *} \mathrm{P}<0.01 \mathrm{vs}\right.$. KO mice). (D) Gene expression at protein level was detected by western blotting and was significantly increased in the KO mice compared with the WT mice at E18.5 and P2 (n=6). (E) Increased mRNA levels of SP-A, SP-B, and SP-C were quantified by reverse transcription-quantitative polymerase chain reaction in E18.5 and P2 lungs of KO mice versus WT mice ( $\mathrm{n}=6$, "P<0.05; ${ }^{* *} \mathrm{P}<0.01 ;{ }^{* * *} \mathrm{P}<0.001$ vs. KO mice). miR, microRNA; SP, surfactant protein; KO, knockout mice; WT, wild-type mice; IOD, integrated optical density. 
by western blotting (E18.5, P2; Fig. 3D). Furthermore, the RT-qPCR analyses of the mRNA levels of SP-A, SP-B and SP-C also demonstrated a significant increase in miR-26a knockout E18.5 and P2 lungs (Fig. 3E). These results supported the hypothesis that knockout of miR-26a induced an increase in PS synthesis.

\section{Discussion}

The fetal lung undergoes extensive physiological and biochemical maturation prior to birth in preparation for its postnatal function as an organ for gas exchange (19). PS, a unique developmentally regulated, phospholipid-rich lipoprotein, is synthesized by AECIIs of the pulmonary alveolus and is stored in organelles termed lamellar bodies $(2,7)$. PS lines the alveoli with a role to decrease surface tension at the air-liquid interface and so facilitate the expansion of the lungs (20). PS is markedly increased during late lung development, and is required for proper lung development and the adaptation to air breathing following birth $(6,12,19)$. Underdevelopment of lung structure and/or any reduction in the ability of AECIIs to produce PS contribute significantly to the morbidity and mortality of infants born prematurely $(1,19,20)$. The present study aimed to explore the potential role of miR-26a in lung formation and PS synthesis at different stages of lung development using a miR-26a double knockout mouse model.

miRNAs have been increasingly recognized to serve crucial roles in the regulation of lung maturation and PS synthesis, including miR-17, miR-20a, miR-106b and miR-127 (21-23). In the authors' previous study, miRNA profiling was performed at three-time points (E16, E19 and E21) of the developing fetal rat lung and the results suggested that miR-26a was upregulated during late lung development, indicating that miR-26a may serve an important role in lung development (9). Previous studies have indicated that miR-26a serves a critical role in tumorigenesis, either as a tumor suppressor or as an oncogenic miRNA, depending on different tumor types, however there have been few studies on the association between miR-26a and lung development (24-27). The authors' previous study identified that the overexpression of miR-26a in AECIIs inhibited PS synthesis by regulating SMAD1-associated bone morphogenic protein (BMP) signaling pathways, which serve essential roles in regulating lung development and function $(2,11,28)$. A miR-26a-1/miR-26a-2 double knockout mouse model was successfully created using the CRISPR/Cas9 system and the homozygous double knockout mice represented an ideal experimental model to analyze the function of miR-26a in vivo. Furthermore, it was identified that PS synthesis and the number of AECIIs were significantly increased in miR-26a knockout mice (17). However, data from in vitro and transgenic animal studies remain lacking. To the best of the authors' knowledge, the present study was the first to explore the effects of miR-26a during different stages of lung development in vitro using knockout mice.

The present study was concerned with three critical time points (E16.5, E18.5 and P2). To characterize the lung formation in miR-26a double knockout mice, the prenatal and neonatal lung morphology at different developmental stages was examined by H\&E staining. At E16.5, lungs progressed to the canalicular stage and epithelial cells were altered to a low cuboidal shape, observed in mutant mice and wild-type mice lungs, however dilated lumens and more aerated area were observed in mutant mice indicating increased maturity of fetal lungs. At the saccular stage, a large number of AECIIs were produced. An increase in the maturation of alveolar structure at E18.5 was observed and there were more AECIIs in double knockout mice lungs at P2, compared with wild-type mice lungs. Lamellar bodies are the intracellular storage form of PS and serve an important role in the production of PS. By using transmission electron microscopy, it was identified that the number of lamellar bodies increased at E18.5 and P2 in mutant mice. In order to further understand the role of miR-26a in PS synthesis, immunohistochemistry, RT-qPCR and western blot analysis were performed to detect the expression of SP-A, SP-B and SP-C, which serve important roles in the metabolism and surface characteristics of PS. mRNA and protein levels of SP-A, SP-B and SP-C increased in mutant mice compared with wild-type mice at the late stages of lung development. These findings indicated that knockout of miR-26a increases the maturation in lung formation and the expression of surfactant-associated proteins, which is crucial for gas exchange following birth.

Although the present study considered the role of miR-26a in lung development in vivo, there remain a number of deficiencies. A previous study identified that miR-26a is selectively expressed in bronchial and alveolar epithelial cells of the murine lung (10). However, the experimental model employed in the present study was not a tissue-specific knockout mice model. A previous in vitro study suggested that miR-26a in fetal rat AECIIs inhibits PS synthesis by regulating SMAD1-associated BMP signal pathways (2), however the mechanism by which miR-26a regulates PS synthesis and lung development in vivo remains to be elucidated and further research is required.

In conclusion, miR-26a serves an essential role during lung development. Notably, the findings of the present study indicated that knockout of miR-26a may promote the maturation in lung formation and increase the expression of surfactant-associated proteins in PS synthesis, which implicated the potential application of miR-26a in the therapy of neonatal respiratory distress.

\section{Acknowledgements}

The authors would like to thank the Nanjing Key Laboratory of Pediatrics for technical assistance.

\section{Funding}

The present study was supported by funding from the National Natural Science Foundation of China (grant no. 81270725).

\section{Availability of data and materials}

The datasets used and/or analyzed during the current study are available from the corresponding author on reasonable request.

\section{Authors' contributions}

YFS, QK and XYZ designed the experiments. YFS, YY, YHZ, JXS and CZ performed experiments, collected the data 
presented in the present study and interpreted results. YFS, QK and XYZ wrote the manuscript.

\section{Ethics approval and consent to participate}

All procedures were approved by the Nanjing Medical University Animal Ethical and Welfare Committee (Nanjing, China).

\section{Consent for publication}

Not applicable.

\section{Competing interests}

The authors declare that they have no competing interests.

\section{References}

1. Martis PC, Whitsett JA, Yan X, Perl AT, Wan HJ and Ikegami M: C/EBPalpha is required for lung maturation at birth Development 133: 1155-1164, 2006.

2. Zhang XQ, Zhang P, Yang Y, Qiu J, Kan Q, Liang HL, Zhou XY and Zhou XG: Regulation of pulmonary surfactant synthesis in fetal rat type II alveolar epithelial cells by microRNA-26a. Pediatr Pulmonol 49: 863-872, 2014.

3. Brasch F, Schimanski Sven, Muhlfeld C, Barlage S, Langmann T, Aslanidis C, Boettcher A, Dada A, Schroten H, Mildenberger E, et al: Alteration of the pulmonary surfactant system in full-term infants with hereditary ABCA3 deficiency. Am J Respir Crit Care Med 174: 571-580, 2006.

4. Gesche J, Fehrenbach H, Koslowski R, Ohler FM, Pynn CJ, Griese M, Poets CF and Bernhard W: rhKGF stimulates lung surfactant production in neonatal rats in vivo. Pediatr Pulmonol 46: 882-895, 2011.

5. Han S and Mallampalli RK: The Role of Surfactant in lung disease and host defense against pulmonary infections. Ann Am Thorac Soc 12: 765-774, 2015

6. Jin J, Li YC, Ren JG, Lam SM, Zhang YD, Hou Y, Zhang XJ, Xu R, Shui GH and Ma RZ: Neonatal respiratory failure with retarded perinatal lung maturation in mice caused by reticulocalbin 3 disruption. Am J Respir Cell Mol Biol 54: 410-423, 2016

7. El-Gendy N, Kaviratna A, Berkland C and Dhar P: Delivery and performance of surfactant replacement therapies to treat pulmonary disorders. Ther Deliv 4: 951-980, 2013.

8. Herriges M and Morrisey EE: Lung development: Orchestrating the generation and regeneration of a complex organ. Development 141: 502-513, 2014.

9. Yang Y, Kai G, Pu XD, Qing K, Guo XR and Zhou XY: Expression profile of microRNAs in fetal lung development of sprague-dawley rats. Int J Mol Med 29: 393-402, 2012.

10. Williams AE, Moschos SA, Perry MM, Barnes PJ and Lindsay MA: Maternally imprinted microRNAs are differentially expressed during mouse and human lung development. Dev Dyn 236: 572-580, 2007.

11. Sun JP, Chen H, Chen C, Whitsett JA, Mishina Y, Bringas P, Jr, Ma JC, Warburton D and Shi W: Prenatal lung epithelial cell-specific abrogation of Alk3-bone morphogenetic protein signaling causes neonatal respiratory distress by disrupting distal airway formation. Am J Pathol 172: 571-582, 2008.
12. Orgeig S, Morrison JL and Daniels CB: Prenatal development of the pulmonary surfactant system and the influence of hypoxia. Respir Physiol Neurobiol 178: 129-145, 2011.

13. Jinek M, Chylinski K, Fonfara I, Hauer M, Doudna JA and Charpentier E: A programmable dual-RNA-guided DNA endonuclease in adaptive bacterial immunity. Science 337: 816-821, 2012.

14. Wiedenheft B, Sternberg SH and Doudna JA: RNA-guided genetic silencing systems in bacteria and archaea. Nature 482: 331-338, 2012.

15. Li D, Qiu Z, Shao Y, Chen Y, Guan Y, Liu M, Li Y, Gao N, Wang L, Lu X, et al: Heritable gene targeting in the mouse and rat using a CRISPR-Cas system. Nat Biotechnol 31: 681-683, 2013.

16. Niu Y, Shen B, Cui Y, Chen Y, Wang J, Wang L, Kang Y, Zhao X, Si W, Li W, et al: Generation of gene-modified cynomolgus monkey via Cas9/RNA-mediated gene targeting in one-cell embryos. Cell 156: 836-843, 2014.

17. Zhang YH, Wu LZ, Liang HL, Yang Y, Qiu J, Kan Q, Zhu W, Ma CL and Zhou XY: Pulmonary surfactant synthesis in miRNA-26a-1/miRNA-26a-2 double knockout mice generated using the CRISPR/Cas9 system. Am J Transl Res 9: 355-365, 2017.

18. Livak KJ and Schmittgen TD: Analysis of relative gene expression data using real-time quantitative PCR and the 2 (-Dalta Dalta C(T)) method. Methods 25: 402-408, 2001.

19. King G, Maker GL, Berryman D, Trengove RD and Cake MH: Role of neuregulin-1beta in dexamethasone-enhanced surfactant synthesis in fetal type II cells. FEBS Lett 588: 975-980, 2014.

20. Smith LJ, McKay KO, Asperen PP, Selvadurai H and Fitzgerald DA: Normal development of the lung and premature birth. Paediatr Respir Rev 11: 135-142, 2010.

21. Carraro G, El-Hashash A, Guidolin D, Tiozzo C, Turcatel G, Young BM, Langhe SP, Bellusci S, Shi W, Parnigotto PP and Warburton D: miR-17 family of microRNAs controls FGF10-mediated embryonic lung epithelial branching morphogenesis through MAPK14 and STAT3 regulation of E-Cadherin distribution. Dev Biol 333: 238-250, 2009.

22. Nana-Sinkam SP, Karsies T, Riscili B, Ezzie M and Piper M: Lung microRNA: From development to disease. Expert Rev Respir Med 3: 373-385, 2009.

23. Bhaskaran M, Wang Y, Zhang HH, Weng TT, Baviskar P, Guo YJ, Gou DM and Liu L: MicroRNA-127 modulates fetal lung development. Physiol Genomics 37: 268-278, 2009.

24. Deng JJ, He MX, Chen LZ, Chen C, Zheng JM and Cai ZL: The loss of miR-26a-mediated post-transcriptional regulation of cyclin E2 in pancreatic cancer cell proliferation and decreased patient survival. PLoS One 8: e76450, 2013.

25. Tan YW, Ge GH, Pan TL, Wen DF, Chen L, Yu XJ, Zhou XB and Gan JH: A serum microRNA panel as potential biomarkers for hepatocellular carcinoma related with hepatitis B virus. PLoS One 9: e107986, 2014.

26. Salvatori B, Iosue I, Mangiavacchi A, Loddo G, Padula F, Chiaretti S, Peragine N, Bozzoni I, Fazi F and Fatica A: The microRNA-26a target E2F7 sustains cell proliferation and inhibits monocytic differentiation of acute myeloid leukemia cells. Cell Death Dis 3: e413, 2012.

27. Ichikawa T, Sato F, Terasawa K, Tsuchiya S, Toi M, Tsujimoto G and Shimizu K: Trastuzumab produces therapeutic actions by upregulating miR-26a and miR-30b in breast cancer cells. PLoS One 7: e31422, 2012.

28. Chen C, Chen H, Sun J, Bringas P Jr, Chen Y, Warburton D and Shi W: Smad1 expression and function during mouse embryonic lung branching morphogenesis. Am J Physiol Lung Cell Mol Physiol 288: L1033-1039, 2005. 